\title{
Lectura con fines investigativos en programas empresariales a distancia con apoyo virtual
}

Methodological strategy for teaching-learning of sports activities through the use of ICT

Diana Esperanza Jiménez Vacca

Programa de Administración de Empresas, Unidad de Ciencias Empresariales, Corporación Universitaria Minuto de Dios, Villavicencio, Colombia.

Correo electrónico: djimenezvac@uniminuto.edu.co

Saúl Eduardo Rojas Gutierrez

Programa de Administración de Empresas, Unidad de Ciencias Empresariales, Corporación Universitaria Minuto de Dios, Villavicencio, Colombia.

Correo electrónico: srojasgutie@uniminuto.edu.co 


\section{Resumen}

El presente artículo es componente estructural del proceso de construcción de las líneas de gestión para el fomento de la lectura con fines investigativos en programas universitarios a distancia con apoyo virtual, sea el caso, los correspondientes a una Unidad de Ciencias Empresariales en Villavicencio (Meta) y los procesos relacionados con las funciones sustantivas, haciendo énfasis en la investigación disciplinar en aplicación de metodologías de grupos focales para la identificación de categorías referidas a lectura, la identificación de propuestas para incentivar actividades en este sentido y la comprensión de la importancia de la lectura en los estudiantes en la modalidad a distancia con apoyo virtual. Desde lo institucional, se identifican aspectos metodológicos en relación con el estudio de las disciplinas, el desarrollo de las actividades de investigación y la relación docenciainvestigación en aplicación de un modelo praxeológico.

Palabras clave: investigación disciplinar, lectura, modalidad distancia, programas empresariales.

\section{Abstract}

This article is a structural component of the process of construction of the management lines for the promotion of reading for research purposes in distance-based university programs with virtual support, be the case, those corresponding to a Business Sciences Unit in Villavicencio (Meta) and the processes related to substantive functions, emphasizing disciplinary research in the application of focus group methodologies for the identification of categories related to reading, the identification of proposals to encourage activities in this regard and the understanding of the importance of reading students in distance learning with virtual support. From the institutional point of view, methodological aspects are identified in relation to the study of disciplines, the development of research activities and the teaching-research relationship in application of a praxeological model.

Keywords: disciplinary research, reading, distance mode, business programs.

\section{Introducción}

El propósito del proyecto "Líneas de Gestión para el fomento de la lectura y la investigación disciplinar en una Unidad de Ciencias Empresariales", es el de realizar la socialización de la información, (1) de la Unidad de Ciencias Empresariales, (2) la investigación disciplinar en la unidad de Ciencias Empresariales relacionada con el modelo praxeológico, (3) la pedagogía y didáctica para la gestión de la lectura con fines investigativos, y (4) identificar líneas de gestión para el fomento de la lectura y la investigación disciplinar; estas acciones son posibilidades en la devolución creativa, información específica para la apropiación del modelo praxeológico y divergencia-confluencia de conceptos en espacios construidos para este efecto (Juliao, 2011); así divulgar la información, desde la cognición distribuida, "se distribuye entre los individuos, se construye socialmente para lograr objetivos comunes en 
una cultura; va más allá del individuo, surge de la actividad compartida"(Díaz Barriga, 2006, p.18).

Por tanto, la gestión crea líneas investigativas, mejora contenidos y currículos, sustenta la gestión de conocimiento y su socialización, e impacta el entorno frente a necesidades diversas. Metodológicamente, el estudio es no experimental, trasversal, descriptivo; y se soporta inicialmente en la técnica de grupos focales, haciendo uso del método de muestreo por conveniencia.

\section{De la Unidad de Ciencias Empresariales}

Desde su surgimiento en Bogotá (1990), con énfasis en desarrollo social (Parra, 2010) y su extension por diferentes regiones del país, en Villavicencio se articula en programas de modalidad a distancia con apoyo virtual, tales como, administración en salud ocupacional, administración de empresas, contaduría pública y administración financiera, a agosto de 2018, la relación de estudiantes muestra 2.379 matriculados, de los cuales $72,3 \%$ son mujeres y $27,7 \%$ son hombres; con relación a la edad, el 9,3\% tienen entre 16 a 19 años, el $68,5 \%$ se encuentra entre 20 a 29 años, igual o mayores de 30 años el 22,2\%. En cuento al estrato socioeconómico, el $76 \%$ pertenecen al estrato 1 y 2 , el $23 \%$ al estrato 3 y tan solo el $1 \%$ a los estratos 4 y 5 .

Para el desarrollo metodológico en grupo focal, se constituyen a manera de pilotaje, grupos de estudiantes que manifiesten de manera voluntaria su interés de participar en el pro- ceso de investigación, por tanto un muestreo a conveniencia se ajusta para para la aplicación en la unidad, a saber, el programa de Administración en Salud Ocupacional: 9 estudiantes, de Contaduría Pública: 12 estudiantes, de Administración Financiera: 10 estudiantes, de Administración de Empresas: 11 estudiantes; de esta manera se construye la base del primer ejercicio que busca obtener información específica de la Unidad de Ciencias Empresariales; la investigación disciplinar en estos programas en cuanto al modelo praxeológico, la pedagogía y didáctica para la gestión de la lectura con fines investigativos y las posibles líneas de gestión para el fomento de la lectura y la investigación disciplinar.

Consecuentemente se solventan prioridades para la investigación principal, como base para el desarrollo posterior de cuatro fases, (1) caracterización de la bibliografía utilizada por los profesores de los cuatro programas según componente disciplinar, y aquellos textos utilizados en los trabajos de grado de los programas entre el periodo 2013-2017; (2) identificación de la relación entre los textos incluidos en la bibliografía de las asignaturas disciplinares frente a las investigaciones clasificadas por líneas de investigación; (3) análisis de información obtenida a partir de entrevistas, encuestas, libros y trabajos de investigación, complementando los temas de investigación disciplinar de interés de profesores y estudiantes; (4) construcción de una propuesta que considere estrategias metodológicas, alineadas al Proyecto Educativo Institucional. 


\section{Investigación disciplinar en los programas de la Unidad de Ciencias Empresariales y el modelo praxeológico}

La investigación se destaca de forma importante en los programas de administración y contaduría, según investigaciones realizadas en los programas mencionados; Saldarriaga, Martínez \& Restrepo (2016), mencionan dos aspectos importantes de la investigación disciplinar en los programas de administración, por una parte, quienes orientan los cursos de formación en investigación, no son administradores, aportando solo aspectos metodológicos y no disciplinares; y por otra parte, la mayoría de las maestrías en administración son profesionalizantes y no investigativas, lo que fortalece el hacer y no el crear. Lo que evidencia, que quienes orientan los cursos transversales de investigación aportan en el componente metodológico, pero no en el disciplinar; y aquellos docentes que imparten en los cursos disciplinares aportan en los componentes disciplinares desde la didáctica, pero es débil en métodos investigativos para generar conocimientos nuevos.

De igual forma, se identifica que las facultades de administración en Colombia, se dividen en dos tipos de facultades: las de mediano desarrollo de investigación, representada en el $61 \%$ de la muestra; y de bajo desarrollo de investigación, con un 39\% de la muestra; y no se identificó un grupo de alto desarrollo (Calderón, Gutiérrez \& Castaño, 2017), lo que muestra un gran reto para las facultades, si se quiere alcanzar un alto desarrollo de la investigación desde los aspectos de recursos, prácticas, estructura y las políticas de la investigación.
Por otra parte, en la investigación contable, se destacan los siguientes aspectos: que sólo se registran 33 grupos de investigación contable frente a los 242 programas contables registrados en el SNIES; los temas de investigación no se centran en la solución de temas administrativos del contexto, sino en la teoría, la epistemología contable y la educación contable (Patiño, et al., 2016). Con referencia a la Unidad de Ciencias Empresariales, que oferta el programa de Contaduría, el modelo de investigación requiere mayor divulgación, apropiación y aplicación por parte de profesores y estudiantes (Trujillo \& López, 2014), siendo aspectos importantes a tener en cuenta para el desarrollo de las líneas de gestión en el presente estudio.

De los programas empresariales objeto de estudio, se resaltan los trabajos de grado, como una modalidad de investigación formativa que fortalece la investigación disciplinar, comprendido en el periodo entre 2013 a 2017, identificando 126 trabajos, de los cuales el 65\% corresponden al programa de Administración de Empresas, seguido de Contaduría Pública con un $21,4 \%$, finalmente los programas de Administración Financiera y Administración en Salud Ocupacional con un $6,8 \%$ cada uno. En relación a los propósitos, la mayoría son planes de negocios (43\%), seguido de herramientas empresariales (36\%), factibilización o viabilización en la creación de empresas (19\%) y finalmente trabajos de grado, cuya finalidad es la gestión en empresas en diferentes áreas funcionales.

Identificando la relación con el modelo pedagógico, a partir del objetivo que contempla "la elaboración, experimentación y validación de modelos de acción, útiles para la gestión de 
la praxis: como forma de formalizar, validar y programar lo que generalmente se hace de modo espontáneo, intuitivo y empírico (Juliao, 2011, p.53); se plantea articulación entre la docencia y la investigación orientada contextualizar la teoría, a validarla con la práctica, este enfoque, contempla las fases como "ver", buscando responder a planteamientos del ¿Qué sucede?; juzgar, que busca identificar ¿Qué puede hacerse?; actuar, donde se define ¿Qué puede hacerse en concreto; y finalmente la fase de devolución creativa, en la cual se indaga sobre ¿Que aprendimos de lo que hacemos?

Para la investigación en observación simple, se resalta la relación docencia - investigación en la fase de juzgar, pues los estudiantes mayormente se cuestionan ¿qué puede hacerse?, al momento de enfrentarse a espacios académicos donde pueden realizar actividades de lectura con fines investigativos, identificando parámetros y herramientas para reflexionar en tal sentido, y soportarse en la teoría o en construcción de postulados, así como lo expone Flores (2016) , cuando afirma, que "si queremos alumnos, gente, sociedades, civilizaciones creativas, que cuenten con la habilidad intrínseca de solucionar los problemas de hoy en día, necesitamos nutrir y verdaderamente enseñar la lectura, la escritura y el pensamiento crítico en las aulas" (p.134); es decir, el fomento de la lectura, como en este caso, con fines investigativos, además de fortalecer competencias investigativas, aporta en el desempeño laboral y el mejoramiento en el rol dentro de la sociedad, acorde con la fase de devolución creativa que permite interactuar la teoría con la práctica, y aprender del ejercicio práctico.

Así mismo, la relación docencia - investigación, contribuye a que la docencia imparta el nuevo conocimiento generado y no se estanque el proceso de aprendizaje; "la investigación universitaria es un proceso de búsqueda de nuevo conocimiento, proceso caracterizado por la creatividad del acto, por la innovación de ideas $\mathrm{y}$, por consiguiente, no está aislado del hecho de la enseñanza" (Zúñiga, 2015, p.16).

Bajo estas líneas, es relevante exponer los niveles de conocimiento en relación a la percepción de los estudiantes sobre cómo se desarrolla la investigación disciplinar desde la docencia y cuáles son sus propuestas para fomentarla, ejercicio aplicado en grupos focales con estudiantes a partir de la pregunta detonante, ¿por qué consideran que es importante la lectura para la investigación disciplinar, y qué aspectos sustentan dicha importancia?

Figura 1. Importancia de la lectura para la investigación disciplinar.

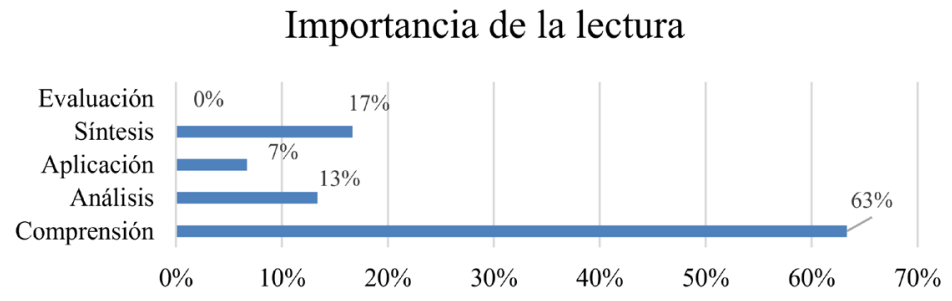

Fuente. Elaboración propia, basada en la taxonomía de Bloom. 
Considerando a Bloom para el análisis, y los seis niveles, conocimiento, comprensión, aplicación, análisis, síntesis y evaluación (Antolín, 2010); en la Figura 1, se observa que la mayoría de las manifestaciones de los estudiantes, se ubican en el nivel de comprensión, puesto que consideran que es importante comprender las diferentes temáticas de lectura, reforzando el conocimiento, aportando al aprendizaje y mejorando la comunicación para el desarrollo profesional; seguido, el nivel de síntesis con un $17 \%$, puesto que consideran que contribuye a desarrollar la capacidad crítica, ayudando a delimitar las temáticas y los fundamentos de la investigación. En tercer lugar, está el nivel de análisis, que permite una fundamentación temática, la identificación de vacíos, y un apoyo en relación a los docentes. Del nivel de conocimiento "evaluación", no se identifica ninguna manifestación en la población participante de la toma de datos.

\section{Pedagogía y didáctica para la gestión de la lectura con fines investigativos}

Los profesores en el quehacer diario de la docencia, han contribuido a fomentar la lectura con fines investigativos, destacando que se presenta, tanto en las actividades realizadas en clase, como en las horas de trabajo independiente o de trabajo autónomo, atendiendo a lo establecido en los lineamientos de la gestión docente y en apropiación de los diferentes aspectos pedagógicos, en el entendido que, "la pedagogía es una disciplina que estudia y propone estrategias para lograr la transición de las personas del estado natural al estado humano" (Flórez, 1994, citado por Cossio, 2014, p.17); al igual que la didáctica general, considerada como "ciencia que trata de la enseñanza escolar en general, bajo cualquier aspecto de normas y principios, y estudia fenómenos y leyes" (Navarro, 2011, p.13).

En el desarrollo del estudio de la gestión de la lectura con fines investigativos, se analiza desde las asignaturas donde los estudiantes reconocen que se viene realizando investigación disciplinar en el componente curricular correspondiente; de igual forma, al hacer uso de diferentes aspectos pedagógicos orientados por los profesores, se observa coincidencia entre lo que se investiga en las aulas y los intereses de investigación; además, de identificar consistencia en el tipo, diseño y enfoque que tiene una mayor utilización en los espacios académicos, acorde a las áreas a las que pertenecen los libros de los que los estudiantes tienen mayor recordación.

Figura 2. Aspectos en los que se ha desarrollado la investigación disciplinar.

\section{Asignaturas según componentes}

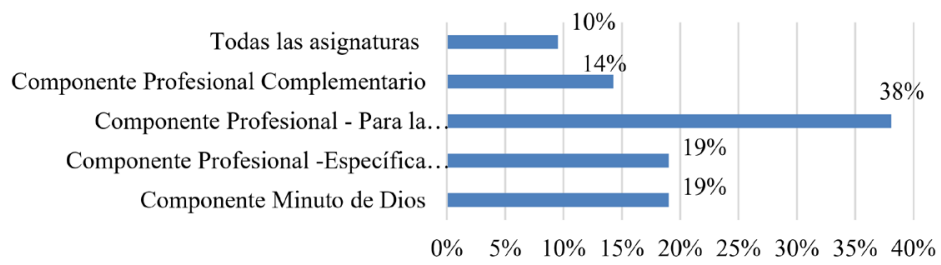

Fuente. Elaboración propia, basada en la estructura curricular del modelo educativo universitario. 
Indudablemente, los procesos investigativos, contribuyen en la gestión de la lectura, por lo que se indaga sobre este aspecto a los estudiantes que hacen parte de los grupos focales (ver Figura 2), de lo que se identifica, respecto del cuestionamiento ¿en qué asignaturas se ha desarrollado la investigación disciplinar?, que se agrupa por componentes curriculares, se logra determinar que el componente profesional en formación para la investigación, se desarrolla con las asignaturas de Investigación Formativa e Introducción a la Investigación, representando el $38 \%$; seguida del componente profesional específico profesional y el compo- nente misional con $19 \%$ cada uno; el componente profesional complementario, constituido principalmente por las prácticas profesionales, opción de grado y electivas, con el 14\%, y finalmente, el $10 \%$, que corresponde a los participantes que manifiestan que en todas las asignaturas se desarrolla la investigación disciplinar.

En la Tabla 1, se presenta los temas investigados por los estudiantes de la Unidad de Ciencias Empresariales, así como que temáticas que son de su interés para futuras investigaciones.

Tabla 1. Temas de investigación realizados y temas de investigación de interés.

\begin{tabular}{|c|c|}
\hline Temas investigados & Temas de investigación de interés \\
\hline \multicolumn{2}{|l|}{ Administración en Salud Ocupacional } \\
\hline $\begin{array}{l}\text { Riesgos labores: en el área operativa, ergonómicos, mecánicos, } \\
\text { físico en el ruido, químicos / Condiciones de iluminación / Enfer- } \\
\text { medades de la voz / Sistema integrado de gestión / Diseño de una } \\
\text { herramienta de formación para el personal con pérdida auditiva } \\
\text { que retoman sus labores / Plan de capacitación para la formación } \\
\text { riesgos laborales. }\end{array}$ & $\begin{array}{l}\text { Sistemas de gestión integral / Plan de capacitación para la forma- } \\
\text { ción riesgos laborales / Hipoacusia / Riesgos ergonómicos en todos } \\
\text { los métodos }\end{array}$ \\
\hline \multicolumn{2}{|l|}{ Contaduría Pública } \\
\hline Cultura / legalidad / Máquinas para reciclar: ecobots & $\begin{array}{l}\text { Aplicación de las NIIF / Auditoria en el sector salud / Análisis conta- } \\
\text { bles de empresas reales / Temas tributarios / Cultura de legalidad en } \\
\text { contabilidad pública / Calidad ISO } 2015 \text { / Análisis financieros / Ética } \\
\text { profesional / Temas laborales / Creación de empresa. }\end{array}$ \\
\hline \multicolumn{2}{|l|}{ Administración Financiera } \\
\hline $\begin{array}{l}\text { Crecimiento de pequeñas empresas / Acceso al crédito estudiantil } \\
\text { / Deserción académica en Administración Financiera / Efectos de la } \\
\text { inflación en población marginal. }\end{array}$ & $\begin{array}{l}\text { Influencia tiene la cultura y las familias sobre los hijos para el } \\
\text { crecimiento y el desarrollo económico de un ciudad, región o país } \\
\text { / Emprendimiento / Formas de invertir }\end{array}$ \\
\hline \multicolumn{2}{|l|}{ Administración de Empresas } \\
\hline $\begin{array}{l}\text { Factores para fortalecer la calidad educativa en la educación supe- } \\
\text { rior / ¿por qué los colombianos no ahorran? / Historia de la legisla- } \\
\text { ción tributaria en Colombia / Procesos de inventarios / Creación } \\
\text { de empresa sobre organización de eventos }\end{array}$ & $\begin{array}{l}\text { Por qué las personas no emprenden o cual es el miedo a empren- } \\
\text { der / Origen y desarrollo de la administración / Bajo interés de } \\
\text { los estudiantes para investigar / Gerencia estratégica en los bancos } \\
\text { / Porque que los estudiantes no continúan con sus proyectos de } \\
\text { grado / Neuromarketing }\end{array}$ \\
\hline
\end{tabular}

Fuente. Elaboración propia.

Al realizar la comparación de los temas investigados por parte de los estudiantes y los temas de interés se puede observar en la Figura 3, la evidencia que los programas de Administración en Salud Ocupacional están investigan- do temas disciplinares y estos coinciden con los de interés. Por otra parte, los programas de Administración Financiera y de Empresas, aunque parte de las investigaciones son en temas disciplinares, estos no coinciden con los 
intereses de investigación. Y finalmente, Contaduría, aunque no menciona que esté realizando investigaciones netamente disciplinares, si tienen claridad en los temas disciplinares que les gustaría investigar.

Figura 3. Comparación entre temas investigados y temas de interés en Unidad de Ciencias Empresariales.

Administración en Salud Ocupacional

Se identifica que tanto los temas investigados como los de interés son muy similares: riesgos laborales, sistemas de gestión integral y planes de formación.

Administración Financiera

Aunque se ha investigado en temas disciplinares como el crédito estudiantil, los temas de interés no coinciden con los temas de interés, pues estos se relacionan con formas de invertir relación entre la cultura y el crecimiento financiero.

\section{Contaduría Pública}

Puesto que los temas investigados no son disciplinares, sin embargo, los temas de interés sí lo son como Aplicación de las NIIF, Temas tributarios y laborales, ética profesional entre otros temas.

\section{Administración de Empresas}

Presenta ciertas diferencias entre los temas investigados y los de interés por investigar, por ejemplo han investigado sobre el ahorro, historia tributaria e inventarios y les interesa la gerencias estratégica y el neuromarketing. Sin embargo, coinside en el tema de emprendimiento.
De los aspectos pedagógicos y didácticos, se indaga si tienen recordación de fuentes entre libros y artículos científicos consultados para realizar una investigación, evidenciando, que no hay una recordación. Sin embargo, se tiene como referente de consulta libros disponibles en la biblioteca institucional. De igual forma, a la pregunta ¿cuántos libros consultan?, se presenta un rango entre 10 a 30 fuentes.

Figura 4. Tipo, diseño y enfoque de las investigaciones promovidas en las clases.

Tipo, diseño y enfoque de la investigaciones promovidas en las clases

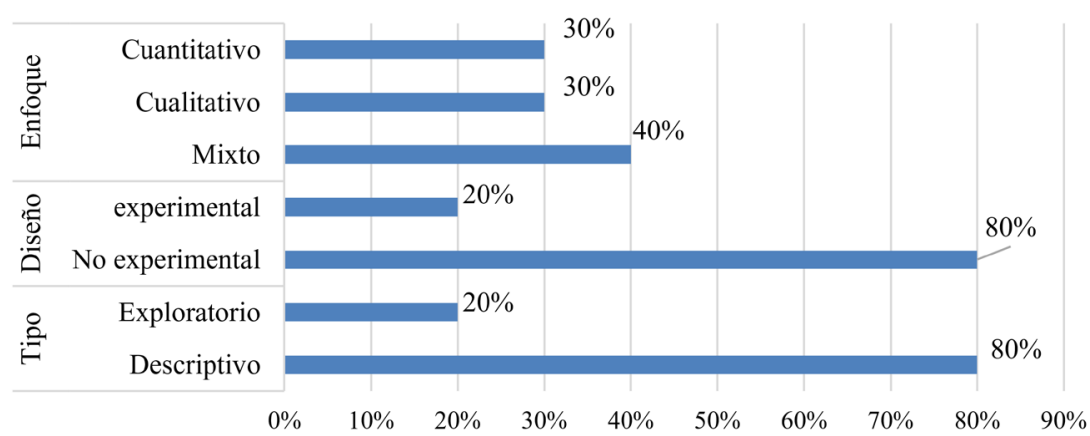


Teniendo en cuenta las investigaciones realizadas desde los espacios académicos, en la Figura 4 se muestra que el $80 \%$ son de tipo descriptivas, mientras que tan solo el $20 \%$ explora- torias; el $80 \%$ tiene un diseño no experimental y el 20\% experimental; con relación al enfoque, el $40 \%$ tienen un enfoque mixto, el 30\% cuantitativo y el $30 \%$ cualitativo.

Figura 5. Áreas de los libros referidos en los cursos para el desarrollo de investigaciones.

Áreas de los libros disciplinares referidos en los cursos para el desarrollo de investigaciones

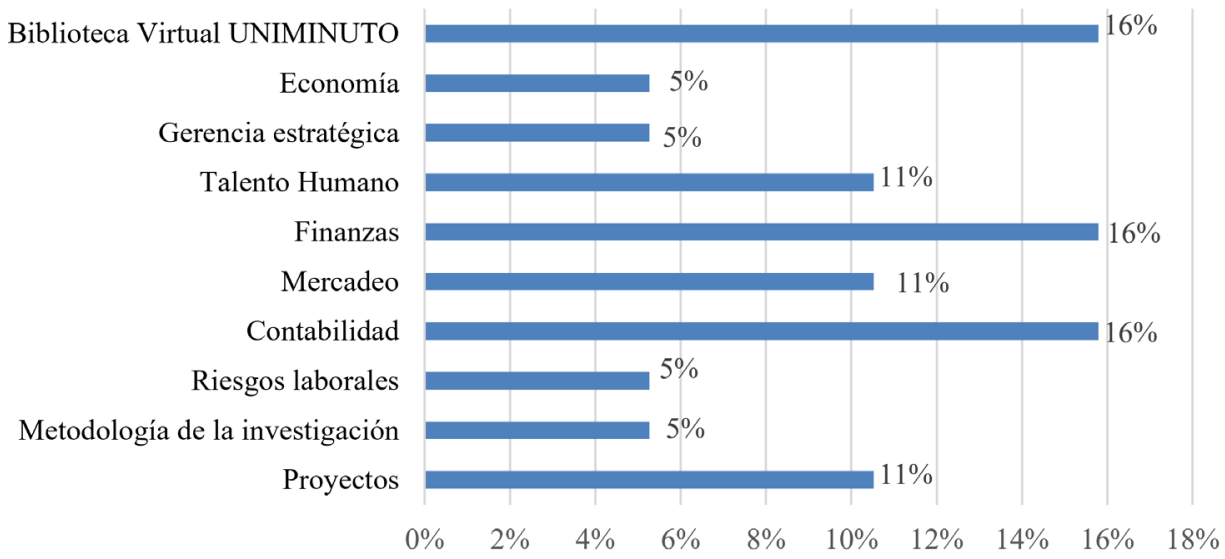

En la Figura 5, se observa que las áreas que mayor recordación, se relacionan con libros disciplinares, de Finanzas (16\%) y Contabilidad (16\%), considerándolo ajustado al programa de Administración Financiera, seguido de Talento Humano, Mercadeo y Proyectos, con un 11\% cada uno, que son áreas funcionales de las empresas, objeto de estudio de los Administradores de Empresas. Finalmente, es de resaltar que el 16\%, no tiene una recordación por un área específica, sino que mencionan la Biblioteca Institucional, como la fuente de consulta para el desarrollo de investigaciones disciplinares.

Además de libros disciplinares, se evidencia la importancia de generar un punto de encuentro entorno a revistas científicas de cada área disciplinar, puesto que no hay una recordación de revistas científicas disciplinares utilizadas en los cursos; solo aquellas publicaciones no científicas como revista Dinero, revista Semana y páginas web.

De igual forma, se les pregunta a los estudiantes acerca de los espacios para reflexionar sobre ¿cómo realizar una investigación?, identificando el que se reconoce la biblioteca virtual y las bases de datos, así como espacios de discusión como semilleros, seminarios y laboratorios.

En relación con los textos de metodología de la investigación para el desarrollo de investigaciones, se destacan los libros de Metodología de la investigación de Hernández Sampieri, Métodos de la investigación e Introducción a la investigación; otros estudiantes manifiestan no recordar nombres de libros; y otros manifiestan que no se documentan con textos, sino que siguen las orientaciones de los profesores o utilizan el internet. 


\section{Líneas de gestión para el fomento de la lectura y la investigación disciplinar}

Figura 6. Propuestas para incentivar la lectura con fines investigativos.

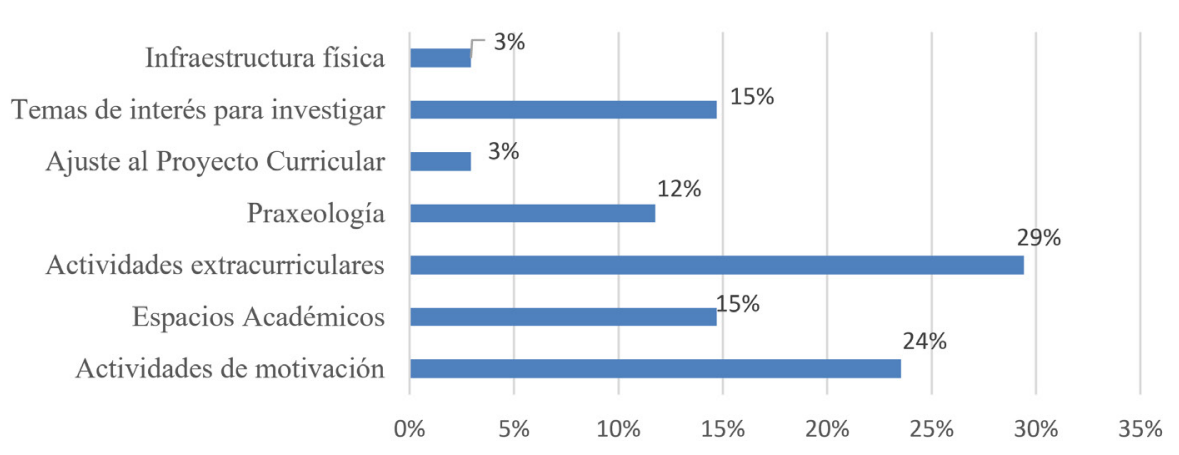

Para identificar las diferentes formar de incentivar la lectura en los estudiantes, con el objeto de desarrollar procesos de investigación, o el estudio de métodos para la investigación, se les indaga sobre las propuestas posibles; presentándose información sobre siete aspectos, en el siguiente orden de participación: el 29\% consideran que se deben realizar actividades extracurriculares como capacitaciones sobre herramientas bibliográficas, ferias de libros y de investigaciones con stands, difusión de las diferentes modalidades de investigación, charlas sobre experiencias exitosas de investigación; el $24 \%$ propuso actividades de motivación como charlas y publicidad sobre la importancia de la lectura, la investigación y sus beneficios; 15\% promueven que los espacios académicos ofrezcan actividades dinámicas para incentivar la lectura y a la investigación; otro $15 \%$ considera que se debe inducir a los estudiantes para que investiguen sobre temas de interés, temas actuales que generen expectativas al estudiante como motivación para la investigación; tan solo el $6 \%$ considera que se deben hacer cambios en el proyecto curricular de los programas y el mejoramiento de la infraestructura física para un mejor acceso a espacios de lectura y de investigación disciplinar.

\section{Conclusiones}

Teniendo en cuenta los objetivos trazados, para la presente ponencia, se puede concluir, primero, que, aunque todos los estudiantes consideran que la lectura es importante, la mayoría no desarrolla procesos cognoscitivos importantes en este sentido, buscando solamente el nivel básico de la comprensión; con el agravante de desconocer el cómo mejorar sus procesos para lograr procesos de mejor desarrollo cognoscitivo, sea síntesis, evaluación, procesos metacognitivos o procesos de autorregulación en aras de consolidar procesos de investigación disciplinar.

Segundo, el desarrollo de la investigación disciplinar desde la docencia, debe contemplar los diferentes tipos o alcances de investi- 
gación, como el explicativo, correlacional y no limitarse solo al tipo descriptivo y exploratorio; requiriéndose un mejoramiento para ampliar el alcance a las investigaciones y con ello a los resultados. De igual forma, se puede concluir que las asignaturas en que los estudiantes reconocen como espacios de investigación, son transversales, pertenecientes al área de investigación y de aspectos misionales, dejando en un tercer plano las asignaturas disciplinares. Pues, es de reconocer que las asignaturas de investigación son las que proporcionan el soporte metodológico, pero son las asignaturas disciplinares, el deber ser del actuar o de devolución creativa, para el aprovechamiento de los recursos para el aprendizaje, para una praxis que articule la teoría con la práctica en el entorno y el reconocimiento del mismo mediante la lectura y la investigación disciplinar.

Los docentes dentro de sus espacios académicos, como en las actividades extracurriculares de discusión, deben generar más acciones para descubrir temas de interés para la investigación de los estudiantes, así también, generar espacios para su desarrollo, pues es determinante para la disminución de las brechas entre los temas investigados y los de interés, permitiendo incrementar la motivación por investigar y el logro de metas de desarrollo en los procesos de aprendizaje; se identifica que las líneas de gestión a contemplar, se soportan, (1) en diseñar unas actividades de sensibilización, con charlas, publicidad donde se muestren los beneficios de la lectura con fines investigativos, así como la investigación disciplinar; (2) actividades extracurriculares que fortalezcan las competencias investigativas como talleres, charlas, foros, ferias; (3), que en los espacios académicos se haga uso diferentes metodologías que acerquen a los estudiantes a la lectura y a la investigación; (4) que independientemente si es un espacio académico o extracurricular, que los estudiantes puedan realizar investigaciones que sean de su interés, pues solo de esta forma se garantizará que las demás actividades mencionadas puedan lograr el fomento de la investigación disciplinar.

\section{Referencias}

Antolín, J. (2010). Taxonomía Cognitiva de Bloom. Recuperado de: https://www.researchgate.net/publication/285057623_Taxonomia_ Cognitiva_de_Bloom

Díaz, F. (2006). Enseñanza situada. México: McGraw-Hill Interamericana.

Calderón, G., Gutiérrez, L. \& Castaño, G. (2017). La investigación en las facultades de administración de Colombia. Revista Lasallista de Investigación, 14(1), 42-55. Recuperado de: http://www.scielo.org.co/pdf/ rlsi/v14n1/1794-4449-rlsi-14-01-00042.pdf

Cossio Moreno, J. (2014). Pedagogía y calidad de la educación: una mirada a la formación del maestro rural. Sophia, 10(1), 14-23. Recuperado de: https://www.redalyc.org/ pdf/4137/413734078002.pdf

Flores, D. (2016). La importancia e impacto de la lectura, redacción y pensamiento crítico de la educación superior. Zona Próxima, 24 (enero-junio), 2016 Recuperado de: http://www.redalyc.org/articulo. oa? $\mathrm{id}=85346806010$ 
Juliao, C. (2011). El enfoque praxeológico. Corporación Universitaria Minuto de Dios Uniminuto. Facultad de Educación (EDU). Departamento de Pedagogía - Escuela de Alta Docencia Bogotá D.C., Colombia. Recuperado de: https://repository.uniminuto.edu/bitstream/handle/10656/1446/ El\%20Enfoque\%20Praxeologico.pdf?sequence $=3 \&$ is Allowed $=y$

Hamui-Sutton, A. \& Varela-Ruiz, M. (2013). La técnica de grupos focales. Investigación en educación médica, 2(5), 55-60. Recuperado de: http://www.scielo.org. $\mathrm{mx} /$ scielo.php?script=sci_arttext $\&$ pi$\mathrm{d}=$ S2007-50572013000100009\&lng=es\&tl$\mathrm{ng}=$

Parra, R. (2010). Avances de la Facultad de Ciencias Empresariales de la Corporación Universitaria Minuto de Dios en sus 18 años de funcionamiento. Revista Gestión \& Desarrollo. Recuperado de: http://revistas. uniminuto.edu/index.php/DYG/article/ view/442

Patiño, R., Valero, G., Zapata, J. \& Díaz, M. (2016). La investigación contable en Colombia. Una aproximación a su comprensión. Teuken Bidikay, 09, 37-54. Recuperado de: http://revistas.elpoli.edu.co/index.php/ teu/article/view/1008

Saldarriaga, J. G., Martínez, J. \& Restrepo, M. L. (2016). La formación en los postgrados en administración: desarrollo de competencias para la investigación. Revista Espacios, 37(10). Recuperado de: http://www. revistaespacios.com/a16v37n10/163710e1. html
Trujillo, J. \& López., J. (2014). La formación para la investigación y su incidencia en la cualificación académica de los estudiantes de contaduría en Colombia. Teuken Bidikay, 05. Recuperado de: https://www. researchgate.net/publication/283509433_ La_formacion_para_la_investigacion_y su_incidencia_en_la_cualificacion_academica_de_los_estudiantes_de_Contaduria_ en_Colombia

Zúñiga, W. (2015). Una perspectiva acerca de la investigación y la docencia universitaria en Colombia. En Clave Social, 4(1). Recuperado de: http://repository.lasallista.edu. co:8080/ojs/index.php/EN-Clave/article/ view/947/894

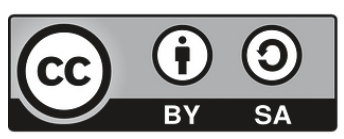

